\title{
Coleopterofauna (Insecta: Coleoptera) coletada em armadilhas com óleo essencial de citronela ou seus componentes no município de Ponta Grossa, Paraná, Brasil
}

\author{
Bruno Piotrovski Begha ${ }^{\bowtie}$, Carlos Henrique Antunes \& Julianne Milléo
}

\section{EntomoBrasilis 11 (2): $78-84$ (2018)}

\begin{abstract}
Resumo. Coleoptera é a ordem mais numerosa e diversificada de Insecta. Possuem diversos tipos de alimentação, sendo que os besouros herbívoros atacam cultivos agrícolas e plantas ornamentais causando prejuízos aos agricultores. Conhecer as espécies que afetam o cultivo local é uma forma de diminuir seu impacto e propor formas de manejo corretas. Os objetivos deste trabalho foram identificar os coleópteros coletados em armadilhas contendo o óleo de citronela ou seus componentes (citronelal, citronelol e geraniol) instaladas em Ponta Grossa, Paraná e, caracterizar a coleopterofauna através de diferentes análises ecológicas. Somente os coleópteros foram identificados e contabilizados. O programa ANAFAU analisou: índices de frequência, abundância, dominância e constância. Pelo PAST avaliou-se a diversidade, equitatividade e similaridade da comunidade atraída pelas armadilhas. Foram coletados 1269 coleópteros, distribuídos em 23 famílias. Diabrotica speciosa (Germar) foi a única espécie superdominante, superabundante, superfrequente e constante ao longo de toda a amostragem, correspondendo a 54,68\% das coletas. Citronela 1\% (25,22\%), citronelol 1\% (25,06\%) representaram 50,28\% das capturas. Citronela $1 \%$ teve a maior diversidade e riqueza de espécies e citronelol $0,5 \%$ a menor. O citronelal $1 \%$ e o citronelol $1 \%$ apresentaram maior similaridade entre as espécies capturadas nestas duas armadilhas. É possível inferir que a distribuição dos coleópteros capturados nos compostos ocorreu devido à presença de moléculas do atrativo também estarem presentes em seus feromônios. O elevado número de $D$. speciosa coletadas confirma o potencial atrativo dos compostos testados que podem vir a auxiliar na redução de pragas agrícolas, diminuindo o uso de agrotóxicos.
\end{abstract}

Palavras-chave: Análises faunísticas; citronelal; citronelol; compostos atrativos; diversidade.

\section{Coleopterofauna (Insecta: Coleoptera) collected in traps with essential oil of citronella or its components in the city of Ponta Grossa, Paraná, Brazil}

\begin{abstract}
Coleoptera is the most numerous and diverse order of Insecta. They have various feeding behaviors, being that herbivorous beetles attack agricultural crops and ornamental plants, causing losses to farmers. Knowing the species that affect the local culture is a way to reduce its impact and to propose correct ways of handling them. The objectives of this research were to identify Coleoptera collected in traps containing citronella oil or its components (citronellal, citronellol and geraniol) installed in Ponta Grossa, Paraná, and to characterize the coleopterofauna through different ecological analysis. Only beetles were identified and counted. The ANAFAU program analyzed: indices of frequency, abundance, dominance and constancy. For PAST, the diversity, equity and similarity of the community attracted by the traps was evaluated. A total of 1269 beetles were collected, distributed in 23 families. Diabrotica speciosa (Germar) was the only superdominant, superabundant, superfrequent and constant species throughout the whole sampling, corresponding to 54.68\% of the captures. Citronella $1 \%$ (25.22\%) and citronellol 1\% (25.06\%) represented 50.28\% of the catches. Citronella $1 \%$ had the highest diversity and species richness and citronellol $0.5 \%$ the lowest. Citronellal $1 \%$ and citronellol $1 \%$ showed greater similarity between the species captured in these two traps. It is possible that the distribution of the Coleoptera captured in the compounds occurred due to the presence of attractive molecules also being present in their pheromones. The high number of $D$. speciosa collected confirms the attractive potential of the tested compounds that may help to reduce agricultural pests, reducing the use of agrochemicals.
\end{abstract}

Keywords: Attractive compounds; citronellal; citronellol; diversity; faunistic analysis.

oleoptera é a ordem mais numerosa e diversificada da classe Insecta, estando presente em todos os continentes, comexceçãodas regiões polares, mostrandoaltaadaptação aos mais variados ambientes e métodos de sobrevivência, incluindo água doce, raízes de árvore ou gramíneas (GulLAN \& Cranston 2010).
Muitas das espécies de coleópteros também são herbívoras, alimentando-se de uma ampla variedade de folhas, flores e raízes de cultivos agrícolas e plantas ornamentais. Juntamente com o fato de serem possíveis transmissores de agentes letais para plantas, uma grande população de coleópteros herbívoros pode causar prejuízos de milhões de dólares aos agricultores (BURCHETT 2001). Portanto, conhecer as espécies que mais afetam o cultivo
Edited by:

Ricardo Andreazze (in memoriam)

Article History:

Received: o9.vii.2017

Accepted: $10 . v .2018$
Corresponding author:

Bruno Piotrovski Begha

乃 bpbegha@gmail.com

No ORCID record
Funding agencies:

¿ Fundação Araucária 
local é uma forma de diminuir seu impacto na agricultura e propor formas de manejo corretas a serem aplicadas em cultivos na região.

A região dos Campos Gerais apresenta rendimentos superiores à média agrícola nacional de produção de grãos, e é pioneira na adoção de novas tecnologias, que visam a incrementar ainda mais os rendimentos obtidos (SCHIMANDEIRo et al. 2006), tornando interessante a busca por métodos de controle mais eficientes e que resultem em um produto de melhor qualidade.

Em 2010, a equipe do Laboratório de Bioecologia de Invertebrados da Universidade Estadual de Ponta Grossa, PR. realizaram testes com oito atrativos preparados a partir de óleos essenciais comerciais, com intuito de propor uma alternativa para o controle de moscas-das-frutas (Diptera: Tephritidae). E, notaram que dos 422 insetos capturados no experimento, 21\% eram da espécie Diabrotica speciosa (Germar), atraídas por citronela (Cymbopogon sp.) e, a partir destes resultados, deu-se continuidade aos experimentos. Pois, o uso do óleo essencial de citronela pode vir a ser uma alternativa viável de um produto natural com eficácia ao controle de insetos-praga, com especial interesse na $D$. speciosa.

Nesse contexto, o presente trabalho teve como objetivos identificar os coleópteros coletados em armadilhas contendo o óleo de citronela e seus principais componentes (citronelal, citronelol e geraniol) instaladas em Ponta Grossa, Paraná; apontar os insetos praga; e, caracterizar a fauna capturada através de diferentes análises ecológicas.

\section{MATERIAL E MÉTODOS}

Os experimentos foram realizados em hortas orgânicas do PréAssentamento Emiliano Zapata no município de Ponta Grossa/ PR. E, de acordo com a disponibilidade das espécies vegetais. Neste trabalho não foi analisada a sazonalidade dos coleópteros devido à intermitência das coletas, totalizando cinco períodos de amostragem que ocorreram em: 2010 (novembro - dezembro), 2011 (março - abril), 2012 (outubro - novembro) e 2013 ( $1^{\mathrm{a}}$ coleta: abril - maio; $2^{\mathrm{a}}$ coleta: novembro - dezembro), estes períodos são referentes a material de projetos anteriores que ainda não haviam sido analisados. A metodologia das amostragens teve o seguinte padrão: os insetos foram coletados por meio de armadilhas feitas de garrafas PET (metodologia adaptada de AgUIAR-MENEZEs et al. 2006), as quais ficavam suspensas por meio de suportes de ferro a $20 \mathrm{~cm}$ do solo. As dez armadilhas, uma para cada composto, foram distribuídas em cultura de couve-chinesa (Brassica rappa L. ssp. pekinensis) ou de abobrinha (Cucurbita sp.). Os atrativos utilizados foram soluções diluídas de óleo essencial de citronela em diferentes concentrações (1,2 e 4\%) e posteriormente, soluções com geraniol, citronelal e citronelol a o,5; 1 e $2 \%$, todas com adição de tensoativo para quebrar a tensão superficial da água. Também foram incluídas armadilhas com água e controle negativo, esta última com água e tensoativo. As dez armadilhas foram testadas durante todos os períodos de coleta.

Os insetos capturados foram montados em alfinetes entomológicos, etiquetados e armazenados em gavetas entomológicas. Após a triagem, apenas os coleópteros foram identificados até o menor nível taxonômico possível com chaves de identificação (ARNETT et al. 2002; Buzzi 2002;). O material do estudo encontra-se depositado na Coleção Entomológica dos Campos Gerais (CEGC) no Laboratório de Bioecologia de Invertebrados da Universidade Estadual de Ponta Grossa (UEPG).

Foram analisados os valores brutos dos insetos coletados por composto em que foi coletado. Os dados foram submetidos ao programa ANAFAU, desenvolvido pela ESALQ (MORAES et al. 2003). E, também foram avaliados os índices de frequência, abundância, dominância e constância, cujas análises foram realizadas com o PAST versão 2.17c (HAMmer et al. 2013): A Diversidade de coleópteros atraída pelas armadilhas foi analisada pelos índices de Shannon-Wiener e Margalef; Para verificar se as espécies capturadas estão distribuídas uniformemente em cada armadilha foi calculado o índice de Equidade; A Similaridade entre as armadilhas com relação a composição de espécies foi analisada pelo coeficiente de Jaccard (baseado em dados de ausência/presença e pelo o índice de Morisita (baseado em dados de abundância).

\section{RESULTADOS E DISCUSSÃO}

Foram coletados 1269 coleópteros, distribuídos em 23 famílias: Chrysomelidae (61,94\%), Staphylinidae (6,15\%), Melyridae (5,36\%), Silvanidae (5,28\%), Coccinellidae (3,47\%), Cantharidae (3,31\%), Mycetophagidae (2,99\%), Phalacridae (2,44\%), Nitidulidae (2,36\%), Scarabaeidae (1,89\%), Carabidae (1,26\%), Histeridae (0,87\%), Curculionidae (0,55\%), Mordellidae (o,55\%), Cryptophagidae (o,16\%), Elateridae (o,16\%), Leiodidae (0,16\%), Lymexylidae (0,16\%), Anobiidae (0,08\%), Ciidae (o,08\%), Cucujidae (o,o8\%), Scydmaenidae (o,o8\%) e os não identificados (0,63\%) (Tabela 1).

O objetivo inicial do projeto foi testar a eficácia do óleo de citronela e seus compostos como atrativos para capturar D. speciosa. E, de acordo com as análises do programa ANAFAU, D. speciosa foi a única espécie que se apresentou como superdominante, superabundante, superfrequente e constante ao longo de todo o período de amostragem, correspondendo a 54,68\% do total das coletas (Figura 1) e confirmando que a espécie está presente na comunidade local e é atraída pelos compostos testados.

A D. speciosa, também conhecida como vaquinha, é um dos principais coleópteros fitófagos considerados como praga em culturas de milho, soja, feijão e arroz, tanto em sua fase larval, ao

Tabela 1. Distribuição e análise faunística das espécies de Coleoptera coletadas em armadilhas contendo óleo de citronela ou seus compostos, entre novembro de 2010 a novembro de 2014, no município de Ponta Grossa-PR.

\begin{tabular}{|c|c|c|c|c|c|c|c|}
\hline Família & Espécie & $\mathbf{N}^{\circ}$ & Coletas & Do & $\mathbf{A b}$ & $\mathbf{F r}$ & Co \\
\hline Anobiidae & Lasiodema sp. & 1 & 1 & ND & $\mathrm{r}$ & $\mathrm{PF}$ & $\mathrm{Z}$ \\
\hline \multirow[t]{5}{*}{ Cantharidae } & Cantharidae sp. & 2 & 2 & ND & $\mathrm{r}$ & $\mathrm{PF}$ & $\mathrm{Z}$ \\
\hline & Chauliognathus sp. 1 & 27 & 10 & $\mathrm{D}$ & $\mathrm{ma}$ & MF & $\mathrm{W}$ \\
\hline & Chauliognathus sp. 2 & 11 & 8 & $\mathrm{D}$ & ma & MF & $\mathrm{W}$ \\
\hline & Chauliognathus sp. 3 & 1 & 1 & ND & $\mathrm{r}$ & $\mathrm{PF}$ & $\mathrm{Z}$ \\
\hline & Chauliognathus sp. 4 & 1 & 1 & ND & $\mathrm{r}$ & $\mathrm{PF}$ & $\mathrm{Z}$ \\
\hline \multirow[t]{3}{*}{ Carabidae } & Axinopalpus sp. & 1 & 1 & ND & $\mathrm{r}$ & $\mathrm{PF}$ & $\mathrm{Z}$ \\
\hline & Bradycellus sp. & 2 & 1 & ND & $\mathrm{r}$ & $\mathrm{PF}$ & $\mathrm{Z}$ \\
\hline & Harpalini sp. & 2 & 2 & ND & $\mathrm{r}$ & $\mathrm{PF}$ & $\mathrm{Z}$ \\
\hline
\end{tabular}


Tabela 1. Continuação...

\begin{tabular}{|c|c|c|c|c|c|c|c|}
\hline Família & Espécie & $\mathbf{N}^{\mathbf{o}}$ & Coletas & Do & $\mathbf{A b}$ & Fr & Co \\
\hline & Lebia sp. 1 & 6 & 5 & $\mathrm{D}$ & $\mathrm{c}$ & $\mathrm{F}$ & $\mathrm{W}$ \\
\hline & Lebia sp. 2 & 4 & 4 & ND & $\mathrm{c}$ & $\mathrm{F}$ & $\mathrm{W}$ \\
\hline & Leptotrachelus sp. & 1 & 1 & ND & $\mathrm{r}$ & $\mathrm{PF}$ & $\mathrm{Z}$ \\
\hline \multirow[t]{25}{*}{ Chrysomelidae } & Acalymma sp. & 9 & 3 & $\mathrm{D}$ & ma & MF & $\mathrm{Y}$ \\
\hline & Bruchus sp. & 1 & 1 & ND & $\mathrm{r}$ & $\mathrm{PF}$ & $\mathrm{Z}$ \\
\hline & Charidotela sexpunctata & 2 & 2 & ND & $\mathrm{r}$ & $\mathrm{PF}$ & $\mathrm{Z}$ \\
\hline & Colaspis sp. 1 & 3 & 3 & ND & d & $\mathrm{PF}$ & $\mathrm{Y}$ \\
\hline & Colaspis sp. 2 & 1 & 1 & ND & $\mathrm{r}$ & $\mathrm{PF}$ & $\mathrm{Z}$ \\
\hline & Colaspis sp. 3 & 3 & 2 & ND & d & $\mathrm{PF}$ & $\mathrm{Z}$ \\
\hline & Crepidodera sp. & 1 & 1 & ND & $\mathrm{r}$ & $\mathrm{PF}$ & $\mathrm{Z}$ \\
\hline & Diabrotica limitata & 6 & 6 & $\mathrm{D}$ & c & $\mathrm{F}$ & $\mathrm{W}$ \\
\hline & Diabrotica sp. 1 & 2 & 2 & ND & $\mathrm{r}$ & $\mathrm{PF}$ & $\mathrm{Z}$ \\
\hline & Diabrotica speciosa & 694 & 26 & SD & sa & $\mathrm{SF}$ & $\mathrm{W}$ \\
\hline & Disonycha sp. & 1 & 1 & ND & $\mathrm{r}$ & $\mathrm{PF}$ & $\mathrm{Z}$ \\
\hline & Donacia sp. & 1 & 1 & ND & $\mathrm{r}$ & $\mathrm{PF}$ & $\mathrm{Z}$ \\
\hline & Epitrix sp. & 1 & 1 & ND & $\mathrm{r}$ & $\mathrm{PF}$ & $\mathrm{Z}$ \\
\hline & Iphimeis dives & 2 & 1 & ND & $\mathrm{r}$ & $\mathrm{PF}$ & $\mathrm{Z}$ \\
\hline & Isotes sp. & 2 & 2 & ND & $\mathrm{r}$ & $\mathrm{PF}$ & $\mathrm{Z}$ \\
\hline & Kuschelina sp. & 1 & 1 & ND & $\mathrm{r}$ & $\mathrm{PF}$ & $\mathrm{Z}$ \\
\hline & Longitarsus sp. & 2 & 2 & ND & $\mathrm{r}$ & $\mathrm{PF}$ & $\mathrm{Z}$ \\
\hline & Luperini sp. & 1 & 1 & ND & $\mathrm{r}$ & $\mathrm{PF}$ & $\mathrm{Z}$ \\
\hline & Microtheca sp. & 5 & 4 & ND & $\mathrm{c}$ & $\mathrm{F}$ & $\mathrm{W}$ \\
\hline & Paranapiacaba duodecimmaculata & 2 & 2 & ND & $\mathrm{r}$ & $\mathrm{PF}$ & $\mathrm{Z}$ \\
\hline & Paranapiacaba sp. & 1 & 1 & ND & $\mathrm{r}$ & $\mathrm{PF}$ & $\mathrm{Z}$ \\
\hline & Percolaspis sp. & 2 & 2 & ND & $\mathrm{r}$ & $\mathrm{PF}$ & $\mathrm{Z}$ \\
\hline & Spintherophyta semiarauta & 27 & 14 & $\mathrm{D}$ & $\mathrm{ma}$ & MF & $\mathrm{W}$ \\
\hline & Systena sp. 1 & 15 & 7 & $\mathrm{D}$ & ma & MF & $\mathrm{W}$ \\
\hline & Systena sp. 2 & 1 & 1 & ND & $\mathrm{r}$ & $\mathrm{PF}$ & $\mathrm{Z}$ \\
\hline Ciidae & Sulcacis sp. & 1 & 1 & ND & $\mathrm{r}$ & $\mathrm{PF}$ & $\mathrm{Z}$ \\
\hline \multirow[t]{13}{*}{ Coccinellidae } & Brumoides sp. & 2 & 2 & ND & $\mathrm{r}$ & $\mathrm{PF}$ & $\mathrm{Z}$ \\
\hline & Coccinellidae sp. & 1 & 1 & ND & $\mathrm{r}$ & $\mathrm{PF}$ & $\mathrm{Z}$ \\
\hline & Coccinella pulchella & 1 & 1 & ND & $\mathrm{r}$ & $\mathrm{PF}$ & $\mathrm{Z}$ \\
\hline & Cycloneda sanguinea & 4 & 4 & ND & $\mathrm{c}$ & $\mathrm{F}$ & $\mathrm{W}$ \\
\hline & Eriopis connexa & 7 & 4 & $\mathrm{D}$ & a & MF & $\mathrm{W}$ \\
\hline & Harmonia axyridis & 7 & 6 & $\mathrm{D}$ & a & MF & $\mathrm{W}$ \\
\hline & Hyperaspis festiva & 2 & 2 & ND & $\mathrm{r}$ & $\mathrm{PF}$ & $\mathrm{Z}$ \\
\hline & Hippodamia convergens & 5 & 3 & ND & $\mathrm{c}$ & $\mathrm{F}$ & $\mathrm{Y}$ \\
\hline & Pseudoazya sp. 1 & 1 & 1 & ND & $\mathrm{r}$ & $\mathrm{PF}$ & $\mathrm{Z}$ \\
\hline & Pseudoazya sp. 2 & 1 & 1 & ND & $\mathrm{r}$ & $\mathrm{PF}$ & $\mathrm{Z}$ \\
\hline & Psyllobora sp. & 5 & 3 & ND & c & $\mathrm{F}$ & $\mathrm{Y}$ \\
\hline & Scymnus sp. 1 & 7 & 5 & $\mathrm{D}$ & a & MF & $\mathrm{W}$ \\
\hline & Scymnus sp. 2 & 1 & 1 & ND & $\mathrm{r}$ & $\mathrm{PF}$ & $\mathrm{Z}$ \\
\hline Cryptophagidae & Atomaria sp. & 2 & 2 & ND & $\mathrm{r}$ & $\mathrm{PF}$ & $\mathrm{Z}$ \\
\hline Cucujidae & Cucujidae sp. & 1 & 1 & ND & $\mathrm{r}$ & $\mathrm{PF}$ & $\mathrm{Z}$ \\
\hline \multirow[t]{5}{*}{ Curculionidae } & Curculionidae sp. & 1 & 1 & ND & $\mathrm{r}$ & $\mathrm{PF}$ & $\mathrm{Z}$ \\
\hline & Madopterini sp. & 2 & 1 & ND & $\mathrm{r}$ & $\mathrm{PF}$ & $\mathrm{Z}$ \\
\hline & Parisoschoenus sp. & 1 & 1 & ND & $\mathrm{r}$ & $\mathrm{PF}$ & $\mathrm{Z}$ \\
\hline & Orthognathus sp. 1 & 2 & 2 & ND & $\mathrm{r}$ & $\mathrm{PF}$ & $\mathrm{Z}$ \\
\hline & Orthognathus sp. 2 & 1 & 1 & ND & $\mathrm{r}$ & $\mathrm{PF}$ & $\mathrm{Z}$ \\
\hline Elateridae & Conoderus sp. & 2 & 2 & ND & $\mathrm{r}$ & $\mathrm{PF}$ & $\mathrm{Z}$ \\
\hline
\end{tabular}


Tabela 1. Continuação...

\begin{tabular}{|c|c|c|c|c|c|c|c|}
\hline Família & Espécie & $\mathbf{N}^{\circ}$ & Coletas & Do & $\mathbf{A b}$ & $\mathbf{F r}$ & Co \\
\hline \multirow[t]{3}{*}{ Histeridae } & Operclipygus sp. & 1 & 1 & ND & $\mathrm{r}$ & $\mathrm{PF}$ & $\mathrm{Z}$ \\
\hline & Phelister sp. 1 & 5 & 4 & ND & $\mathrm{c}$ & $\mathrm{F}$ & $\mathrm{W}$ \\
\hline & Phelister sp. 2 & 5 & 2 & ND & $\mathrm{c}$ & $\mathrm{F}$ & $\mathrm{Z}$ \\
\hline Leiodidae & Agathidium sp. & 2 & 2 & ND & $\mathrm{r}$ & $\mathrm{PF}$ & $\mathrm{Z}$ \\
\hline Lymexylidae & Atractocerus sp. & 2 & 2 & ND & $\mathrm{r}$ & $\mathrm{PF}$ & $\mathrm{Z}$ \\
\hline Melyridae & Astylus variegatus & 68 & 7 & $\mathrm{D}$ & ma & MF & $\mathrm{W}$ \\
\hline \multirow[t]{3}{*}{ Mordellidae } & Mordella sp. 1 & 2 & 2 & ND & $\mathrm{r}$ & $\mathrm{PF}$ & $\mathrm{Z}$ \\
\hline & Mordella sp. 2 & 1 & 1 & ND & $\mathrm{r}$ & $\mathrm{PF}$ & $\mathrm{Z}$ \\
\hline & Mordellaria sp. & 4 & 3 & ND & $\mathrm{c}$ & $\mathrm{F}$ & $\mathrm{Y}$ \\
\hline \multirow[t]{4}{*}{ Mycetophagidae } & Litargus tetraspilotus & 38 & 8 & $\mathrm{D}$ & $\mathrm{ma}$ & MF & $\mathrm{W}$ \\
\hline & Mycetophagidae sp 1 & 3 & 1 & ND & $\mathrm{d}$ & $\mathrm{PF}$ & $\mathrm{Z}$ \\
\hline & Mycetophagidae sp. 2 & 22 & 4 & $\mathrm{D}$ & ma & MF & $\mathrm{W}$ \\
\hline & Mycetophagidae sp. 3 & 3 & 2 & ND & $\mathrm{d}$ & $\mathrm{PF}$ & $\mathrm{Z}$ \\
\hline \multirow[t]{6}{*}{ Nitidulidae } & Carpophilus sp. & 11 & 4 & $\mathrm{D}$ & ma & MF & W \\
\hline & Colopterus sp. 1 & 2 & 2 & ND & $\mathrm{r}$ & $\mathrm{PF}$ & $\mathrm{Z}$ \\
\hline & Colopterus sp. 2 & 2 & 1 & ND & $\mathrm{r}$ & $\mathrm{PF}$ & $\mathrm{Z}$ \\
\hline & Conotelus sp. & 12 & 6 & $\mathrm{D}$ & ma & MF & $\mathrm{W}$ \\
\hline & Cryptarcha sp. & 2 & 2 & ND & $\mathrm{r}$ & $\mathrm{PF}$ & $\mathrm{Z}$ \\
\hline & Lobiopa sp. & 1 & 1 & ND & $\mathrm{r}$ & $\mathrm{PF}$ & $\mathrm{Z}$ \\
\hline \multirow[t]{3}{*}{ Phalacridae } & Litochropus sp. & 1 & 1 & ND & $\mathrm{r}$ & $\mathrm{PF}$ & $\mathrm{Z}$ \\
\hline & Phalacropsis sp. 1 & 1 & 1 & ND & $\mathrm{r}$ & $\mathrm{PF}$ & $\mathrm{Z}$ \\
\hline & Phalacropsis sp. 2 & 1 & 1 & ND & $\mathrm{r}$ & $\mathrm{PF}$ & $\mathrm{Z}$ \\
\hline \multirow[t]{8}{*}{ Scarabaeidae } & Coprophanaeus sp. & 1 & 1 & ND & $\mathrm{r}$ & $\mathrm{PF}$ & $\mathrm{Z}$ \\
\hline & Macraspis sp. & 1 & 1 & ND & $\mathrm{r}$ & $\mathrm{PF}$ & $\mathrm{Z}$ \\
\hline & Macrodactylus sp. & 8 & 3 & $\mathrm{D}$ & ma & MF & $\mathrm{Y}$ \\
\hline & Melolonthinae sp. 1 & 1 & 1 & ND & $\mathrm{r}$ & $\mathrm{PF}$ & $\mathrm{Z}$ \\
\hline & Melolonthinae sp. 2 & 8 & 3 & $\mathrm{D}$ & ma & MF & $\mathrm{Y}$ \\
\hline & Melolonthinae sp. 3 & 2 & 2 & ND & $\mathrm{r}$ & $\mathrm{PF}$ & $\mathrm{Z}$ \\
\hline & Melolonthinae sp. 4 & 1 & 1 & ND & $\mathrm{r}$ & $\mathrm{PF}$ & $\mathrm{Z}$ \\
\hline & Melolonthinae sp. 5 & 2 & 2 & ND & $\mathrm{r}$ & $\mathrm{PF}$ & $\mathrm{Z}$ \\
\hline Scydmaenidae & Euconnus sp. & 1 & 1 & ND & $\mathrm{r}$ & $\mathrm{PF}$ & $\mathrm{Z}$ \\
\hline Silvanidae & Monanus sp. & 67 & 7 & $\mathrm{D}$ & ma & MF & W \\
\hline \multirow[t]{19}{*}{ Staphylinidae } & Aleocharinae sp. 1 & 13 & 8 & $\mathrm{D}$ & $\mathrm{ma}$ & MF & $\mathrm{W}$ \\
\hline & Aleocharinae sp. 2 & 8 & 6 & $\mathrm{D}$ & ma & MF & $\mathrm{W}$ \\
\hline & Aleocharinae sp. 3 & 1 & 1 & ND & $\mathrm{r}$ & $\mathrm{PF}$ & $\mathrm{Z}$ \\
\hline & Aleocharinae sp. 4 & 3 & 2 & ND & $\mathrm{d}$ & $\mathrm{PF}$ & $\mathrm{Z}$ \\
\hline & Aleocharinae sp. 5 & 7 & 5 & $\mathrm{D}$ & a & MF & $\mathrm{W}$ \\
\hline & Aleocharinae sp. 6 & 1 & 1 & ND & $\mathrm{r}$ & $\mathrm{PF}$ & $\mathrm{Z}$ \\
\hline & Anotylus sp. & 19 & 8 & $\mathrm{D}$ & ma & MF & W \\
\hline & Atrecus sp. & 2 & 2 & ND & $\mathrm{r}$ & $\mathrm{PF}$ & $\mathrm{Z}$ \\
\hline & Belonuchus sp. 1 & 3 & 3 & ND & $\mathrm{d}$ & $\mathrm{PF}$ & $\mathrm{Y}$ \\
\hline & Belonuchus sp. 2 & 1 & 1 & ND & $\mathrm{r}$ & $\mathrm{PF}$ & $\mathrm{Z}$ \\
\hline & Bryoporus sp. & 4 & 3 & ND & $\mathrm{c}$ & $\mathrm{F}$ & $\mathrm{Y}$ \\
\hline & Clavilispinus sp. & 1 & 1 & ND & $\mathrm{r}$ & $\mathrm{PF}$ & $\mathrm{Z}$ \\
\hline & Lathrobium sp. 1 & 1 & 1 & ND & $\mathrm{r}$ & $\mathrm{PF}$ & $\mathrm{Z}$ \\
\hline & Lathrobium sp. 2 & 1 & 1 & ND & $\mathrm{r}$ & $\mathrm{PF}$ & $\mathrm{Z}$ \\
\hline & Lissohypnus sp. & 2 & 2 & ND & $\mathrm{r}$ & $\mathrm{PF}$ & $\mathrm{Z}$ \\
\hline & Lithocharis sp. & 2 & 1 & ND & $\mathrm{r}$ & $\mathrm{PF}$ & $\mathrm{Z}$ \\
\hline & Oxytelus sp. & 7 & 2 & $\mathrm{D}$ & $\mathrm{a}$ & MF & $\mathrm{Z}$ \\
\hline & Parothius sp. & 1 & 1 & ND & $\mathrm{r}$ & $\mathrm{PF}$ & $\mathrm{Z}$ \\
\hline & Xanthopygus sp. & 1 & 1 & ND & $\mathrm{r}$ & $\mathrm{PF}$ & $\mathrm{Z}$ \\
\hline
\end{tabular}


Tabela 1. Continuação...

\begin{tabular}{|c|c|c|c|c|c|c|c|}
\hline Família & Espécie & $\mathbf{N}^{\circ}$ & Coletas & Do & $\mathbf{A b}$ & $\mathbf{F r}$ & Co \\
\hline \multirow[t]{6}{*}{ Não identificado } & NI sp. 1 & 2 & 2 & ND & $\mathrm{r}$ & $\mathrm{PF}$ & $\mathrm{Z}$ \\
\hline & NI sp. 2 & 1 & 1 & ND & $\mathrm{r}$ & $\mathrm{PF}$ & $\mathrm{Z}$ \\
\hline & NI sp. 3 & 2 & 2 & ND & $\mathrm{r}$ & $\mathrm{PF}$ & $\mathrm{Z}$ \\
\hline & NI sp. 4 & 1 & 1 & ND & $\mathrm{r}$ & $\mathrm{PF}$ & $\mathrm{Z}$ \\
\hline & NI sp. 5 & 1 & 1 & ND & $\mathrm{r}$ & $\mathrm{PF}$ & $\mathrm{Z}$ \\
\hline & NI sp. 6 & 1 & 1 & ND & $\mathrm{r}$ & $\mathrm{PF}$ & $\mathrm{Z}$ \\
\hline
\end{tabular}

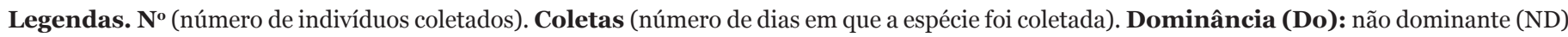
- spp cuja frequência é menor que o limite de dominância, dominante (D) - spp cuja frequência é maior que o limite de dominância, superdominante (SD). Abundância (Ab): raro ( $\mathrm{r})-\mathrm{n}^{\mathrm{o}}$ de indivíduos menor que o limite inferior do IC, a $1 \%$ de probabilidade, disperso (d) - $\mathrm{n}^{\mathrm{o}}$ de indivíduos situado entre os limites inferiores do IC,a $5 \%$ e $1 \%$ de probabilidade, comum (c) - no de indivíduos situado dentro do IC, a $5 \%$ de probabilidade, abundante (a) $-\mathrm{n}^{0}$ de indivíduos situado entre os limites superiores do IC, a $5 \%$ e $1 \%$ de probabilidade, muito abundante (ma) - $\mathrm{n}^{0}$ de indivíduos maior que o limite superior do IC a $5 \%$ de probabilidade, superabundante (sa). Frequência (Fr): pouco frequente (PF), frequente (F), muito frequente (MF), superfrequente (SF). Constância (Co): constante (W) - mais de 50\% das coletas, acessória (Y) - entre 25 a 50\% das coletas; acidental (Z) - menos de $25 \%$ das coletas.

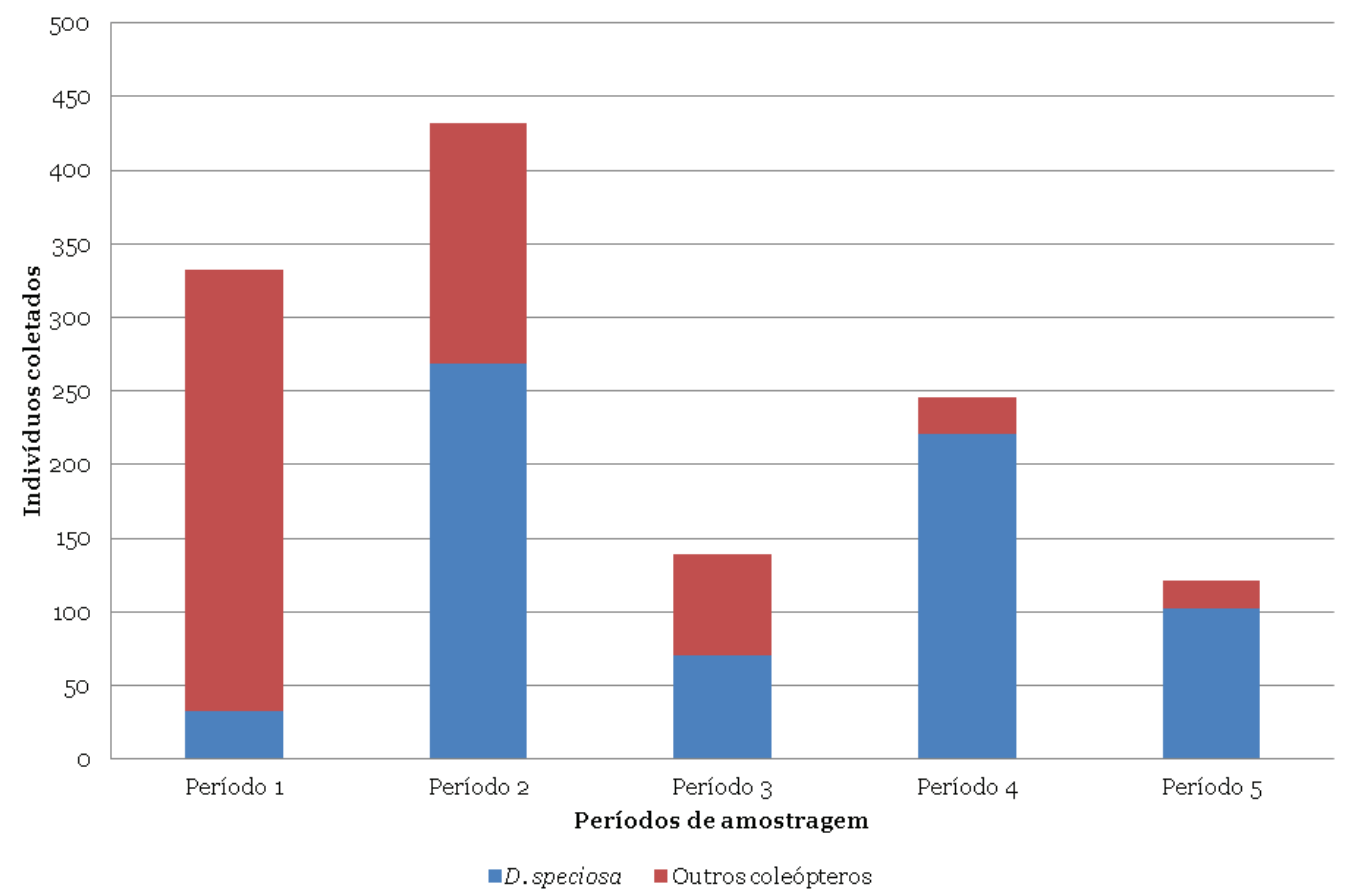

Figura 1. Representatividade de Diabrotica speciosa (Germar) em relação aos outros coleópteros coletados por armadilhas contendo óleo de citronela ou seus compostos, entre novembro de 2010 a novembro de 2014, no município de Ponta Grossa-PR.

se alimentar de raízes, quanto na fase adulta, ao se alimentarem das folhas (VIANA 2010).

As 23 espécies [Astylus variegatus (Germar), Monanus sp., Litargus tetraspilotus LeConte, Chauliognathus sp. 1, Spintherophyta semiarauta (Klug), Mycetophagidae sp. 2, Anotylus sp., Systena sp. 1, Aleocharinae sp. 1, Conotelus sp., Carpophilus sp., Chauliognathus sp. 2, Acalymma sp., Aleocharinae sp. 2, Macrodactylus sp., Nefoncerus sp., Aleocharinae sp. 5, Eriopis connexa (Germar), Harmonia axyridis (Pallas), Scymnus sp. 1, Oxytelus sp., Diabrotica limitata Baly, e Lebia sp. 1] se apresentaram como dominantes, muito abundantes, muito frequentes e constantes, correspondendo a $32,30 \%$ das coletas. Entre estas, A. variegatus (CAMARGO et al. 2001) , S. semiarauta (ZAWADNEAK et al. 2011), Systena sp. (SCHALK et al. 1986), Conotelus sp. (РотіN et al. 2016), Carpophilus sp. (MARINI et al. 2013), Chauliognathus sp. (GorRi et al. 2015), Acalymma sp. (YARDim et al. 2006), Macrodactylus sp. (Williams et al. 2000) e D. limitata (WALSH 2003) são potenciais pragas agrícolas, sendo interessante o controle dessas espécies.

A. variegatus, Melyridae, foi a segunda espécie mais coletada compreendendo 5,35\% das coletas com 68 indivíduos, mostrando uma preferência pelo geraniol 1\% (34 indivíduos). Na fase adulta, o A. variegatus se alimenta de pólen, porém sua larva se alimenta de sementes e brotos, no solo, principalmente de milho sendo considerada uma praga agrícola (VENTURA et al. 2007). O geraniol é um dos compostos de óleos essenciais utilizados por plantas para gerar o odor atrativo em suas flores (SANGwAN et al. 2001). Portanto, é possível que o A. variegatus tenha identificado o odor como uma fonte de alimento em potencial e tenha sido atraído por esta armadilha.

As 92 espécies restantes, correspondendo a 13,02\% das coletas, foram classificadas como não dominantes, raras, pouco frequentes e acidentais.

Em relação ao potencial atrativo dos compostos testados, o número de besouros capturados teve a seguinte distribuição: óleo de citronela $1 \%(25,22 \%)$, citronelol $1 \%(25,06 \%)$, citronela $2 \%(15,52 \%)$, geraniol $1 \%(9,69 \%)$, citronelal $1 \%$ (7,01\%), água (5,36\%), controle negativo (4,81\%), citronelol 2\% (2,92\%), citronelol $0,5 \%(2,44 \%)$ e citronela $4 \%(1,97 \%)$ (Tabela 2$)$.

As moléculas dos compostos testados nestas armadilhas podem ser semelhantes as moléculas presentes nos feromônios de determinadas espécies. Segundo REDdy \& Guerrero (2004), insetos comumente utilizam de compostos voláteis de plantas adquiridos durante a alimentação para sintetizar os próprios feromônios sexuais, e SUZUKi et al. (1983), conseguiu replicar feromônios de besouro castanho [Tribolium castaneum (Herbst)] a partir de componentes do óleo de citronela, mostrando que 
Tabela 2. Número de coleópteros coletados por armadilhas contendo óleo de citronela ou seus compostos, entre novembro de 2010 a novembro de 2014, no município de Ponta Grossa-PR.

\begin{tabular}{|c|c|c|c|c|c|c|c|c|c|c|c|}
\hline \multirow{2}{*}{ Família } & \multirow{2}{*}{ Espécie } & \multicolumn{10}{|c|}{ Compostos } \\
\hline & & Ci 1\% & Ci $2 \%$ & $\mathrm{Ci} 4 \%$ & Ca $1 \%$ & Ge $1 \%$ & Co o,5\% & Co $1 \%$ & Co $2 \%$ & $\mathbf{C N}$ & Ag \\
\hline Anobiidae & Lasiodema sp. & o & o & $\mathrm{o}$ & $\mathrm{o}$ & $\mathrm{o}$ & $\mathrm{o}$ & o & o & 1 & o \\
\hline \multirow[t]{5}{*}{ Cantharidae } & Cantharidae sp. & o & 2 & o & o & o & o & o & o & o & o \\
\hline & Chauliognathus sp. 1 & 8 & 7 & o & 3 & 2 & $\mathrm{o}$ & 2 & o & 2 & 3 \\
\hline & Chauliognathus sp. 2 & o & 3 & o & 0 & 2 & o & 3 & o & 1 & 2 \\
\hline & Chauliognathus sp. 3 & o & o & o & $\mathrm{O}$ & o & $\mathrm{O}$ & o & $\mathrm{o}$ & o & 1 \\
\hline & Chauliognathus sp. 4 & o & o & o & o & 1 & o & o & o & o & o \\
\hline \multirow[t]{6}{*}{ Carabidae } & Axinopalpus sp. & o & o & o & 1 & o & o & o & o & o & 0 \\
\hline & Bradycellus sp. & 1 & 1 & o & o & o & o & o & o & o & o \\
\hline & Harpalini sp. & o & o & o & 1 & o & o & 1 & o & o & o \\
\hline & Lebia sp. 1 & 2 & o & 0 & 2 & o & o & 1 & o & o & 1 \\
\hline & Lebia sp. 2 & o & o & 2 & o & $\mathrm{o}$ & o & 2 & o & o & o \\
\hline & Leptotrachelus sp. & o & o & 0 & 1 & o & o & o & o & o & o \\
\hline \multirow[t]{25}{*}{ Chrysomelidae } & Acalymma sp. & o & o & o & 2 & 3 & o & o & o & 4 & o \\
\hline & Bruchus sp. & o & o & o & 0 & o & o & o & o & 1 & o \\
\hline & Charidotela sexpunctata & o & o & o & o & o & $\mathrm{o}$ & o & 1 & 1 & o \\
\hline & Colaspis sp. 1 & 3 & 0 & o & 0 & o & o & o & o & o & o \\
\hline & Colaspis sp. 2 & o & o & o & o & o & o & o & o & o & 1 \\
\hline & Colaspis sp. 3 & 1 & o & o & 0 & 2 & o & o & o & o & 0 \\
\hline & Crepidodera sp. & o & 1 & o & o & $\mathrm{o}$ & $\mathrm{o}$ & o & o & o & $\mathrm{o}$ \\
\hline & Diabrotica limitata & 5 & 1 & o & 0 & o & o & o & o & o & o \\
\hline & Diabrotica sp. 1 & 2 & o & o & o & $\mathrm{o}$ & $\mathrm{O}$ & o & o & o & o \\
\hline & Diabrotica speciosa & 138 & 110 & 6 & 45 & 43 & 30 & 227 & 34 & 25 & 36 \\
\hline & Disonycha sp. & o & 1 & o & $\mathrm{o}$ & $\mathrm{o}$ & $\mathrm{o}$ & o & o & o & o \\
\hline & Donacia sp. & 1 & o & o & o & o & o & o & o & o & o \\
\hline & Epitrix sp. & 1 & o & 0 & o & o & o & o & o & o & o \\
\hline & Iphimeis dives & o & o & o & o & o & o & o & o & 1 & 1 \\
\hline & Isotes $\mathrm{sp}$. & 1 & o & o & o & o & o & o & o & 1 & o \\
\hline & Kuschelina sp. & o & o & o & o & o & o & 1 & o & o & 0 \\
\hline & Longitarsus sp. & o & 1 & o & 1 & o & o & o & o & o & o \\
\hline & Luperini sp. & o & 1 & o & o & o & o & o & o & o & o \\
\hline & Microtheca sp. & 1 & o & 0 & 1 & 2 & o & o & o & o & 1 \\
\hline & Paranapiacaba duodecimmaculata & 1 & o & o & o & o & 1 & o & o & o & o \\
\hline & Paranapiacaba sp. & o & 1 & $\mathrm{o}$ & 0 & o & o & o & o & o & o \\
\hline & Percolaspis sp. & 1 & o & o & o & o & o & 1 & o & o & o \\
\hline & Spintherophyta semiarauta & 4 & o & o & 1 & o & o & 15 & 2 & 4 & 1 \\
\hline & Systena sp. 1 & 8 & 1 & 1 & 2 & 1 & o & 1 & o & o & 1 \\
\hline & Systena sp. 2 & o & 1 & o & o & o & o & o & o & o & o \\
\hline Ciidae & Sulcacis sp. & o & o & 0 & 0 & 1 & o & o & 0 & o & 0 \\
\hline \multirow[t]{9}{*}{ Coccinellidae } & Brumoides sp. & o & 1 & o & $\mathrm{O}$ & 1 & $\mathrm{O}$ & o & o & o & o \\
\hline & Coccinellidae sp. & o & o & o & o & o & o & o & o & 1 & o \\
\hline & Coccinella pulchella & o & o & o & o & 1 & $\mathrm{o}$ & o & o & o & $\mathrm{o}$ \\
\hline & Cycloneda sanguinea & 1 & o & 0 & o & 1 & o & 1 & o & 1 & o \\
\hline & Eriopis connexa & 3 & o & o & o & 1 & $\mathrm{O}$ & 2 & $\mathrm{o}$ & $\mathrm{o}$ & 1 \\
\hline & Harmonia axyridis & 1 & 1 & o & o & o & o & 1 & o & 1 & 3 \\
\hline & Hyperaspis festiva & o & 1 & o & o & 1 & o & o & o & o & o \\
\hline & Hippodamia convergens & 2 & o & o & o & 0 & o & o & o & 1 & 2 \\
\hline & Pseudoazya sp. 1 & $\mathrm{O}$ & 1 & 0 & 0 & 0 & 0 & 0 & o & o & o \\
\hline
\end{tabular}


Tabela 2. Continuação...

\begin{tabular}{|c|c|c|c|c|c|c|c|c|c|c|c|}
\hline \multirow{2}{*}{ Família } & \multirow{2}{*}{ Espécie } & \multicolumn{10}{|c|}{ Compostos } \\
\hline & & Ci 1\% & Ci $2 \%$ & $\mathrm{Ci} 4 \%$ & Ca 1\% & Ge $1 \%$ & Co $0,5 \%$ & Co $1 \%$ & Co $2 \%$ & CN & Ag \\
\hline & Pseudoazya sp. 2 & 0 & o & 0 & 0 & 1 & 0 & o & o & 0 & o \\
\hline & Psyllobora sp. & 2 & o & o & 1 & 1 & o & 1 & o & o & o \\
\hline & Scymnus sp. 1 & 2 & 2 & 0 & 1 & 1 & 0 & 1 & o & 0 & o \\
\hline & Scymmus sp. 2 & 1 & o & o & o & o & o & o & o & o & o \\
\hline Cryptophagidae & Atomaria sp. & 1 & 1 & o & o & 0 & o & 0 & o & o & o \\
\hline Cucujidae & Cucujidae sp. & o & 0 & 0 & o & 1 & $\mathrm{o}$ & o & o & o & o \\
\hline \multirow[t]{5}{*}{ Curculionidae } & Curculionidae sp. & 0 & 1 & o & 0 & 0 & o & 0 & 0 & 0 & o \\
\hline & Madopterini sp. & 1 & 1 & o & o & o & $\mathrm{o}$ & o & o & o & o \\
\hline & Orthognathus sp. 1 & 0 & 1 & o & o & o & o & o & o & o & 1 \\
\hline & Orthognathus sp. 2 & o & o & o & o & o & o & o & o & o & 1 \\
\hline & Parisoschoenus sp. & 0 & 0 & 0 & 0 & 0 & 0 & 0 & 0 & 0 & 1 \\
\hline Elateridae & Conoderus sp. & o & 0 & o & 1 & o & o & 1 & o & 0 & o \\
\hline \multirow[t]{3}{*}{ Histeridae } & Operclipygus sp. & 1 & 0 & 0 & o & o & o & o & o & o & o \\
\hline & Phelister sp. 1 & 3 & 1 & o & o & o & o & 1 & o & o & o \\
\hline & Phelister sp. 2 & 1 & 3 & o & 1 & o & 0 & o & o & o & o \\
\hline Leiodidae & Agathidium sp. & 2 & o & o & o & o & o & o & o & o & o \\
\hline Lymexylidae & Atractocerus sp. & 2 & 0 & 0 & o & o & 0 & o & o & o & o \\
\hline Melyridae & Astylus variegatus & 22 & o & o & o & 34 & o & 11 & o & 1 & o \\
\hline \multirow[t]{3}{*}{ Mordellidae } & Mordella sp. 1 & o & 1 & o & o & o & o & 1 & o & o & o \\
\hline & Mordella sp. 2 & o & o & o & o & o & o & o & o & 1 & o \\
\hline & Mordellaria & 3 & o & o & o & o & o & 0 & o & o & 1 \\
\hline \multirow[t]{4}{*}{ Mycetophagidae } & Litargus tetraspilotus & 11 & 5 & 1 & 7 & 4 & 0 & 7 & o & o & 3 \\
\hline & Mycetophagidae sp. 1 & 3 & 0 & o & o & 0 & $\mathrm{o}$ & o & o & 0 & o \\
\hline & Mycetophagidae sp. 2 & o & o & 4 & 1 & o & 0 & 7 & o & 3 & 4 \\
\hline & Mycetophagidae sp. 3 & 2 & o & o & 1 & o & o & o & o & o & o \\
\hline \multirow[t]{6}{*}{ Nitidulidae } & Carpophilus sp. & 8 & 2 & o & o & o & $\mathrm{o}$ & 1 & o & o & o \\
\hline & Colopterus sp. 1 & o & o & 1 & o & o & o & 1 & o & o & o \\
\hline & Colopterus sp. 2 & 2 & o & o & o & o & o & o & o & o & o \\
\hline & Conotelus sp. & 2 & 2 & o & 1 & 3 & o & 1 & o & 0 & o \\
\hline & Cryptarcha sp. & o & o & o & o & o & o & o & o & o & o \\
\hline & Lobiopa sp. & o & o & 0 & o & 0 & o & 1 & o & 0 & o \\
\hline \multirow[t]{3}{*}{ Phalacridae } & Litochropus sp. & o & o & o & o & o & 0 & 0 & o & o & o \\
\hline & Phalacropsis sp. 1 & o & 1 & o & o & 0 & o & 0 & o & o & o \\
\hline & Phalacropsis sp. 2 & o & o & o & 0 & 1 & 0 & 0 & o & o & o \\
\hline \multirow[t]{8}{*}{ Scarabaeidae } & Coprophanaeus sp. & 0 & 0 & o & 1 & o & o & o & o & o & 0 \\
\hline & Macraspis sp. & o & o & o & o & 1 & o & o & o & o & o \\
\hline & Macrodactylus sp. & 0 & o & 1 & o & o & o & 0 & o & 7 & o \\
\hline & Melolonthinae sp. 1 & 0 & 0 & o & o & 0 & $\mathrm{o}$ & 0 & o & 1 & o \\
\hline & Melolonthinae sp. 2 & 0 & 3 & 2 & 1 & 3 & 0 & 1 & 0 & 1 & 1 \\
\hline & Melolonthinae sp. 3 & o & o & o & o & 1 & 0 & 0 & o & $\mathrm{o}$ & 1 \\
\hline & Melolonthinae sp. 4 & o & o & 0 & o & o & o & 1 & o & o & o \\
\hline & Melolonthinae sp. 5 & o & 2 & o & o & o & o & o & o & o & o \\
\hline Scydmaenidae & Euconnus sp. & o & 1 & o & o & o & o & o & o & o & o \\
\hline Silvanidae & Monanus sp. & 20 & 18 & 2 & 10 & 5 & 0 & 12 & o & o & o \\
\hline \multirow[t]{3}{*}{ Staphylinidae } & Aleocharinae sp. 1 & 3 & 1 & 2 & 1 & 1 & 0 & 2 & o & 3 & o \\
\hline & Aleocharinae sp. 2 & 2 & 4 & 1 & o & 1 & o & 0 & o & o & o \\
\hline & Aleocharinae sp. 3 & 1 & o & 0 & 0 & o & o & o & 0 & o & o \\
\hline
\end{tabular}


Tabela 2. Continuação...

\begin{tabular}{|c|c|c|c|c|c|c|c|c|c|c|c|}
\hline \multirow{2}{*}{ Família } & \multirow{2}{*}{ Espécie } & \multicolumn{10}{|c|}{ Compostos } \\
\hline & & Ci 1\% & Ci $2 \%$ & $\mathrm{Ci} 4 \%$ & Ca 1\% & Ge $1 \%$ & Co o, $5 \%$ & Co $1 \%$ & Co $2 \%$ & $\mathbf{C N}$ & Ag \\
\hline & Aleocharinae sp. 4 & 2 & o & o & o & o & o & 1 & o & o & o \\
\hline & Aleocharinae sp. 5 & 2 & o & o & o & 2 & o & 1 & o & 1 & 1 \\
\hline & Aleocharinae sp. 6 & o & 1 & o & o & o & o & o & o & o & o \\
\hline & Anotylus sp. & 11 & 3 & 1 & o & o & o & 4 & o & o & o \\
\hline & Atrecus sp. & 1 & o & o & o & o & o & 1 & o & o & o \\
\hline & Belonuchus sp. 1 & 2 & 1 & o & 0 & o & o & o & o & o & o \\
\hline & Belonuchus sp. 2 & o & 1 & o & o & o & o & o & o & o & o \\
\hline & Bryoporus sp. & 3 & 1 & o & o & 1 & o & o & o & 1 & o \\
\hline & Clavilispinus sp. & 1 & o & o & o & o & o & o & o & o & o \\
\hline & Lathrobium sp. 1 & o & 1 & o & o & o & o & o & o & o & o \\
\hline & Lathrobium sp. 2 & 1 & o & o & o & o & o & o & o & o & o \\
\hline & Lissohypnus sp. & 1 & o & 1 & o & o & o & o & o & o & o \\
\hline & Lithocharis sp. & o & o & o & o & o & o & 2 & o & o & o \\
\hline & Oxytelus sp. & 1 & 4 & o & 2 & o & o & o & o & o & o \\
\hline & Parothius sp. & 1 & o & o & o & o & o & o & o & o & o \\
\hline & Xanthopygus sp. & o & o & o & 1 & o & o & o & o & o & o \\
\hline \multirow[t]{6}{*}{ Não identificado } & NI sp. 1 & 1 & o & o & o & o & o & 1 & o & o & o \\
\hline & NI sp. 2 & 1 & o & o & o & o & o & o & o & o & o \\
\hline & NI sp. 3 & 1 & o & o & 0 & $\mathrm{o}$ & $\mathrm{O}$ & 1 & o & o & o \\
\hline & NI sp. 4 & 1 & o & o & 1 & o & o & o & o & o & o \\
\hline & NI sp. 5 & o & o & o & o & o & o & 1 & o & o & o \\
\hline & NI sp. 6 & 1 & o & o & o & o & o & o & o & o & 0 \\
\hline Total de coletados & & 320 & 197 & 25 & 89 & 123 & 31 & 318 & 37 & 61 & 68 \\
\hline Total de espécies & & 58 & 42 & 13 & 26 & 30 & 2 & 37 & 3 & 23 & 22 \\
\hline
\end{tabular}

Legendas. Citronela 1\% (Ci 1\%), Citronela 2\% (Ci 2\%), Citronela 4\% (Ci 4\%), Citronelal (Ca 1\%), Geraniol 1\% (Ge 1\%), Citronelol o,5\% (Co o,5\%), Citronelol 1\% (Co 1\%), Citronelol 2\% (Co 2\%), Controle negativo (CN), Água (Ag).

os coleópteros aqui capturados podem estar utilizando os componentes do óleo essencial da citronela como precursor para seus feromônios. Isso poderia explicar o interesse de uma espécie por determinado composto.

Em relação à diversidade de espécies capturadas pelos diferentes compostos, obteve-se a seguinte sequência: citronela $1 \%>$ citronela $2 \%>$ citronelol $1 \%>$ geraniol $1 \%>$ citronelal $1 \%$ $>$ controle negativo > água > citronela $4 \%>$ citronelol $2 \%>$ citronelol $0,5 \%$.

Como observado anteriormente pela equipe do laboratório durante estudos que também utilizaram compostos derivados de citronela como atrativo para insetos, a família mais representativa foi Chrysomelidae e a espécie mais coletada foi $D$. speciosa, representando 54,68\% das coletas com 694 indivíduos, mostrando preferência pelo citronelol 1\% (227), citronela $1 \%$ (138) e citronela $2 \%(110)$.

O índice de Shannon-Wiener variou de 2,632 a 0,1425 na sequência: citronela $1 \%>$ controle negativo > citronela $4 \%>$ geraniol $1 \%>$ citronelal $1 \%>$ citronela $2 \%>$ água > citronelol $1 \%$ $>$ citronelol $2 \%>$ citronelol o,5\% (Tabela 2).

A diversidade entre os compostos teve um aumento gradual indicando uma diversidade relativamente alta, com exceção de citronelol 1\%, citronelol 2\%, citronelol o,5\% que foram fortemente menos diversos que os outros devido ao grande número de $D$. speciosa coletadas.

Pelo índice de Margalef, valores inferiores a 2,0 foram considerados como compostos com de baixa riqueza e valores superiores 5,0 foram considerados como compostos de alta riqueza. A variação entre compostos foi de 9,931 a 0,2912 (Tabela 2 ), obtendo a sequência citronela $1 \%>$ citronela $2 \%>$ citronelol $1 \%>$ geraniol $1 \%>$ citronelal $1 \%>$ negativo $>$ água $>$ citronela $4 \%$ $>$ citronelol $2 \%>$ citronelol $0,5 \%$. A citronela $1 \%$ acabou tendo uma maior riqueza, possivelmente indicando que uma grande quantidade de táxons deve utilizar citronela na composição de seus feromônios, seja como constituinte ou precursor.

O índice de equitatividade variou de 0,2056 (citronelol 0,5\%) a 0,9143 (citronela 4\%) (Tabela 3). Valores baixos significam compostos com poucas espécies dominantes em meio a muitas espécies raras e valores altos significam a presença de muitas espécies dominantes (MAGURRAN 2003), ou seja, a medida que o valor aumenta, as espécies capturadas por cada composto estão distribuídas de forma mais igualitária.

Pelo coeficiente de Jaccard foram formados dois agrupamentos de compostos no dendrograma A, sendo um subgrupo apenas com o citronelol 0,5\% e citronelol $2 \%$, e o outro subgrupo maior com os demais compostos. O citronelal $1 \%$ e o citronelol $1 \%$ apresentaram maior similaridade entre as espécies capturadas nestas duas armadilhas (Figura 2A).

O dendrograma de similaridade obtido pelo índice de Morisita mostrou vários agrupamentos. Sendo que a composição das espécies capturadas pela armadilha com citronela $4 \%$ mais distinta das demais, seguida pelo geraniol 1\%. Os compostos com citronelol o,5\% e $2 \%$ foram os agrupamentos mais similares, juntos com citronelol $1 \%$. A citronela $2 \%$ e citronelal $1 \%$ se mostraram mais similares e próximos da água e citronela $1 \%$ 
(Figura 2B). Os agrupamentos formados indicaram que os compostos semelhantes terão maior semelhança na estrutura da comunidade de besouros.

O presente projeto conseguiu identificar as espécies componentes da comunidade de coleópteros de Ponta Grossa, Paraná, assim como foi possível observar a distribuição dos insetos perante os diferentes compostos atrativos testados.

Verificou-se que um grande número de coleópteros, considerados praga de espécies vegetais, foram atraídos pelos compostos testados e em especial a $D$. speciosa.
O êxito na utilização de armadilhas com compostos atrativos naturais em plantios significa uma redução no uso de agrotóxicos, auxiliando na preservação do solo e melhorando a qualidade do produto. As formulações a base do óleo essencial de citronela podem vir a colaborar com outras técnicas para redução de pragas agrícolas, pois além do baixo custo dos compostos são facilmente encontrados no mercado.

Sugerem-se futuras pesquisas para investigar se existe relação entre os feromônios atrativos das espécies e os componentes do óleo essencial de citronela.

Tabela 3. Valores obtidos pelos índices de Shannon-Wiener, Margalef e equitatividade das espécies de Coleoptera coletadas em armadilhas contendo óleo de citronela ou seus compostos, entre novembro de 2010 a novembro de 2014, no município de Ponta Grossa-PR.

\begin{tabular}{lccc}
\hline \multirow{2}{*}{ Composto } & & Índice & Equitatividade \\
\cline { 2 - 4 } & Shannon & Margalef & 0,6481 \\
\hline Citronela 1\% & 2,632 & 9,931 & 0,5688 \\
Citronela 2\% & 2,126 & 7,760 & 0,9143 \\
\hline Citronela 4\% & 2,345 & 3,728 & 0,6533 \\
\hline Citronelal 1\% & 2,129 & 5,542 & 0,6690 \\
\hline Geraniol 1\% & 2,275 & 6,026 & 0,2056 \\
Citronelol o,5\% & 0,142 & 0,291 & 0,4107 \\
\hline Citronelol 1\% & 1,483 & 6,241 & 0,3031 \\
Citronelol 2\% & 0,333 & 0,553 & 0,7625 \\
Controle negativo & 2,391 & 5,290 & 0,6647 \\
\hline Água & 2,055 & 4,977 & \\
\hline
\end{tabular}
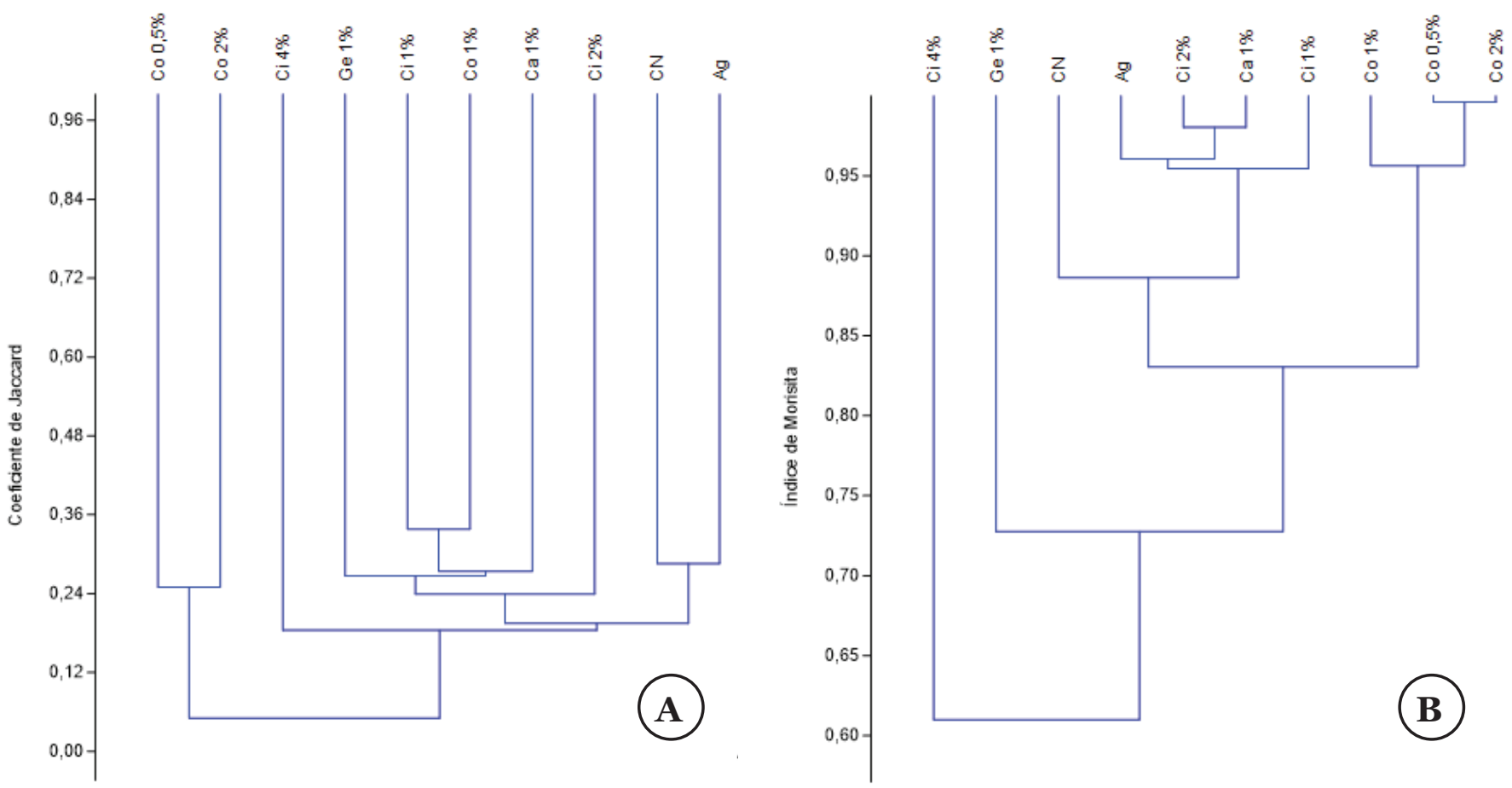

Figura 2. Dendrograma de similaridade da comunidade de Coleoptera coletadas em armadilhas contendo óleo de citronela ou seus compostos, entre novembro de 2010 a novembro de 2014, no município de Ponta Grossa-PR. A) Coeficiente de Jaccard. B) Índice de Morisita.

\section{AGRADECIMENTOS}

À Fundação Araucária pela bolsa de Iniciação Científica cedida ao primeiro autor para a realização desse projeto. À empresa BioFragane Essências \& Fragrâncias pela doação da matéria prima utilizada nos experimentos.

\section{REFERÊNCIAS}

Aguiar-Menezes, E.L.; J.F. Souza; S.A.S. Souza; M.R. Leal; J.R. Costa \& E.B. Menezes, 2010. Armadilha PET para captura de adultos de moscas-das-frutas em pomares comerciais e domésticos. Circular Técnica, 16: 8. Disponível em: <http://https://www.infoteca.cnptia.embrapa.br/handle/ doc/623026 >. [Acesso em: 20.vii.2017].

Arnett, R., M. Thomas, P. Skelley \& J. Frank, 2002. American Beetles, Volume II: Polyphaga: Scarabaeoidea through Curculionoidea. $1^{\text {a }}$ Ed., Boca Raton, CRC Press. 88op.

Burchett, A., 2011. Operation rootworm: Can biotechnology beat the billion-dollar bug? Farm Journal, 125: 16-18.

Buzzi, Z., 2002. Entomologia didática. $6^{\mathrm{a}}$ Ed, Curitiba, Editora UFPR. 579 p. 
Camargo, A.J.A. \& R.F. Amabile, 2001. Identificação das principais pragas do girassol na região CentroOeste. Comunicado Técnico, 50: 4. Disponível em: <http://https://www.embrapa.br/busca-de-publicacoes/-/ publicacao/554106/identificacao-das-principais-pragas-dogirassol-na-regiao-centro-oeste>. [Acesso em: 25.iv.2018].

Gorri, J.E.R., R.C. Pereira, F.M. Alves, F.L. Fernandes, Í.W. Silva \& M.E.S. Fernandes, 2015. Toxicity Effect of Three Insecticides on Important Pests and Predators in Tomato Plants. Agricultural Science, 3: 1-12. DOI: https://doi.org/10.12735/as.v3i1po1.

Gullan, P. \& P. Cranston, 2010. The Insects: An Outline of Entomology. $4^{\text {a }}$ Ed., Hoboken, Wiley-Blackwell. 583 p.

Hammer, Ø, D.A.T. Harper \& P. D. Ryan, 2013. PAST: Paleontological Statistics software package for education and data analysis. Disponível em: <http://https://folk.uio.no/ ohammer/past>.

Magurran, A.E., 2003. Measuring Biological Diversity. $1^{\mathrm{a}}$ Ed., Malden, Blackwell Publishing. 264 p.

Marini, F., F. Mutinelli, F. Montarsi, A.R. Cline, E. Gatti \& P. Audisio, 2013. First report in Italy of the dusky sap beetle, Carpophilus lugubris, a new potential pest for Europe. Journal of Pest Science, 86: 157-160. DOI: https://doi.org/10.1007/s10340-013-0479-9.

Moraes, R.C.B., M.L. Haddad, S.S. Neto \& A.E.L. Reyes, 2003 ANAFAU - Software para análise faunística. Disponível em: <http://www.esalq.usp.br/departamentos/lea/anafau/ anafau.php $>$.

Potin, D.M., G.S. Andrade, R.Z. Pereira \& S.M. Kassab, 2016. Conotelus sp. (Coleoptera: Nitidulidae), a New Insect Pest of Passion Fruit in the Amazon Biome. Florida Entomology, 99: 580-582. DOI: https://doi.org/10.1653/024.099.0345.

Reddy, V.P.G., A. Guerrero. 2004. Interactions of insect pheromones and plant semiochemicals. Trends in Plant Science, 9: 253-261. DOI: https://doi.org/10.1016/j. tplants.2004.03.009.

Sangwan, N.S., A.H.A. Farooqi, F. Shabin \& R.S. Sangwan, 2001. Regulation of essential oil production in plants. Plant Growth Regulation, 34: 3-21.

Schalk, J.M., J.K. Peterson, A. Jones, P.D. Dukes \& M.W.J. Walter, 1986. The anatomy of sweet potato periderm and its relationship to wireworm, Diabrotica systena resistance. Journal of Agricultural Entomology, 3: 350-356.
Schimandeiro, A., P.H.W. Neto, L.M. Gimenez, M.J. Colet \& P.W . Garbuio, 2006. Distribuição longitudinal de plantas de milho (Zea mays L.) na região dos Campos Gerais, Paraná. Ciência Rural, 36: 977-980. DOI: https://doi.org/10.1590/s010384782006000300040.

Suzuki, T., J. Ozaki \& R. Sugawara, 1983. Synthesis of Optically Active Aggregation Pheromone Analogues of the Red Flour Beetle, Tribolium castaneum. Agricultural and Biological Chemistry, 47: 869-875. DOI: https://doi.org/10.1080/000 21369.1983.10865715.

Ventura, M.U., T. Pereira, D.H. Nunes \& I.C. Arruda, 2007. Attraction of Astylus variegatus (Germ.) (Coleoptera: Melyridae) by volatile floral attractants. Scientia Agricola, 64: 305-307. DOI: https://doi.org/10.1590/s010390162007000300016.

Viana, P.A., 2010. Manejo de Diabrotica speciosa na Cultura do Milho. Circular Técnica, 141: 6. Disponível em: <http://https://www.embrapa.br/busca-de-publicacoes/-/ publicacao/875601/manejo-de-diabrotica-speciosa-nacultura-do-milho $>$. [Acesso em: 21.vii.2017].

Walsh, G.C., 2003. Host Range and Reproductive Traits of Diabrotica speciosa (Germar) and Diabrotica viridula (F.) (Coleoptera: Chrysomelidae), Two Species of South American Pest Rootworms, with Notes on Other Species of Diabroticina. Environmental Entomology, 32: 276-285. DOI: https://doi. org/10.1603/0046-225x-32.2.276.

Williams, R.N., D.S. Fickle, T.P. McGovern \& M.G. Klein, 2000. Development of an Attractant for the Scarab Pest Macrodactylus subspinosus (Coleoptera: Scarabaeidae). Journal of Economic Entomology, 93: 1480-1484. DOI: https://doi.org/10.1603/0022-0493-93.5.1480.

Yardim, E.N., N.Q. Arancon, C.A. Edwards, T.J. Oliver \& R.J. Byrne, 2006. Suppression of tomato hornworm (Manduca quinquemaculata) and cucumber beetles (Acalymma vittatum and Diabotrica undecimpunctata) populations and damage by vermicomposts. Pedobiologia, 50: 23-29. DOI: https://doi.org/10.1016/j.pedobi.2005.09.001.

Zawadneak, M., G.H.R. Neto, J.M. Schuber \& H.A. Parchen, 2011. First Record of Spintherophyta semiaurata (Klug) (Coleoptera: Chrysomelidae) Damaging Strawberry Flowers. Neotropical Entomology, 40: 407-408. DOI: https://doi.org/10.1590/s1519-566x2011000300017.

\section{Suggestion citation:}

Begha, B.P., C.H. Antunes \& J. Milléo, 2018. Coleopterofauna (Insecta: Coleoptera) coletada em armadilhas com óleo essencial de citronela ou seus componentes no município de Ponta Grossa, Paraná, Brasil. EntomoBrasilis, 11 (2): 78-84.

Available on: doi:10.12741/ebrasilis.v11i2.734
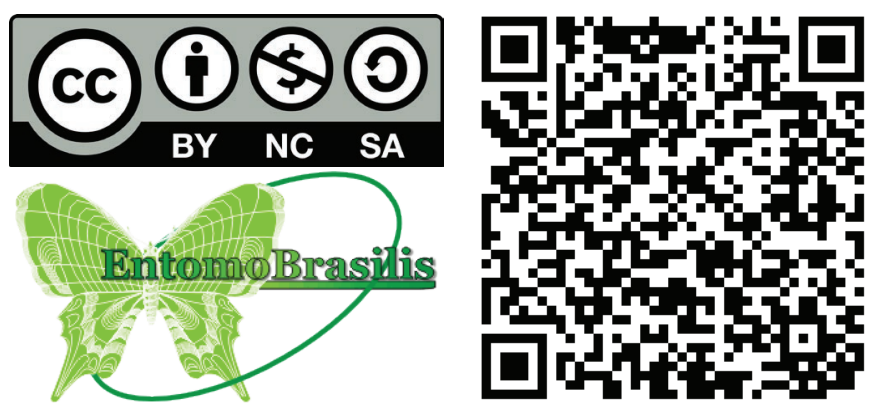